\title{
Quantum Fluids of Self-Assembled Chains of Polar Molecules
}

\section{Citation}

Wang, Daw-Wei, Mikhail D. Lukin, and Eugene Demler. 2006. "Quantum Fluids of SelfAssembled Chains of Polar Molecules." Physical Review Letters 97 (18) (November 3). doi:10.1103/physrevlett.97.180413.

\section{Published Version}

doi:10.1103/PhysRevLett.97.180413

\section{Permanent link}

http://nrs.harvard.edu/urn-3:HUL.InstRepos:27893055

\section{Terms of Use}

This article was downloaded from Harvard University's DASH repository, and is made available under the terms and conditions applicable to Other Posted Material, as set forth at http:// nrs.harvard.edu/urn-3:HUL.InstRepos:dash.current.terms-of-use\#LAA

\section{Share Your Story}

The Harvard community has made this article openly available.

Please share how this access benefits you. Submit a story.

Accessibility 


\title{
Quantum Fluids of Self-Assembled Chains of Polar Molecules
}

\author{
Daw-Wei Wang, ${ }^{1}$ Mikhail D. Lukin, ${ }^{2}$ and Eugene Demler ${ }^{2}$ \\ ${ }^{1}$ Physics Department, National Tsing-Hua University, Hsinchu, Taiwan, Republic of China \\ ${ }^{2}$ Physics Department, Harvard University, Cambridge, Massachusetts 02183, USA
}

(Received 10 August 2006; published 3 November 2006)

\begin{abstract}
We study polar molecules in a stack of strongly confined pancake traps. When dipole moments point perpendicular to the planes of the traps and are sufficiently strong, the system is stable against collapse but attractive interaction between molecules in different layers leads to the formation of dipolar chains, analogously to the chaining phenomenon in classical rheological electro- and magnetofluids. We analyze properties of the resulting quantum liquid of dipolar chains and show that only the longest chains undergo Bose-Einstein condensation with a strongly reduced condensation temperature. We discuss several experimental methods for studying chains of polar molecules.
\end{abstract}

DOI: 10.1103/PhysRevLett.97.180413

Recent progress in trapping and cooling of chromium atoms [1] and polar molecules [2] opened new directions for studying quantum systems with dipole interactions. Manifestations of dipolar interactions have been observed in the density profiles of the chromium condensate [3] and predicted in the dispersion of the Bogoliubov mode [4]. Exotic many-body states arising from the anisotropy and long-range character of dipolar interactions have also been theoretically proposed $[5,6]$. An important aspect of dipolar systems is the attractive part of the interaction for dipoles aligned head to tail, which can lead to the system collapse [7]. To circumvent this problem, most theoretical analysis focused on two- and one-dimensional systems with dipolar moments perpendicular to the plane (axis) of the sample, in which case the attractive part of the interaction is absent.

In this Letter we consider polar molecules in a threedimensional trap which was divided into a stack of twodimensional (2D) pancakes by applying a strong onedimensional optical lattice [8] [see Fig. 1(a)]. When dipolar moments are polarized in the direction perpendicular to the planes, the intralayer interaction is purely repulsive [6]. The attractive interaction, however, is present for dipoles located on top of each other in different layers. The system collapse in the direction of attractive interaction is prevented by the strong optical potential. In the remainder of this Letter we focus on electric dipolar interactions between polar molecules, but our conclusions should also apply to magnetic dipolar atoms [1].

Our main observation is that beyond a certain critical value of the dipole moments, attractive interaction between molecules in different layers can bind them into chains. Longer chains appear first and have a stronger binding energy (see Fig. 2). At finite temperature there is a competition between entropy that favors shorter chains and energy which is minimized for longer chains. This leads to a nonmonotonic dependence of the distribution function on the length of the chains (see Fig. 3). This behavior of the dipolar chains liquid (DCL) strongly resembles rheological electro- and magnetofluids [9]. In both cases the dipolar
PACS numbers: 05.30. $-\mathrm{d}$, 03.75.Hh, 64.60. $-\mathrm{i}, 67.90 .+\mathrm{z}$

interaction leads to self-organization of elementary objects (nanoscale grains in rheological fluids and molecules in our case) into chains of varying length. In the rheological fluids several finite temperature phase transitions have been predicted theoretically or numerically [10]. The chaining phenomena can also be directly imaged in a $2 \mathrm{D}$ thin film [11]. The new feature of DCL in polar molecule systems is the possibility of Bose-Einstein condensation of chains at low temperatures. We find that the longest chains condense first and that in systems with many layers the condensation temperature is strongly suppressed compared to the transition temperature of individual molecules in one layer (inset to Fig. 3). Such suppression can be understood by observing that the presence of chains of different lengths reduces the number of dipoles available for the longest chains and hence limits the number of particles available for the condensation. Finally, we discuss implications of our results for experiments.

We start by investigating the structure of a single chain in a multilayered system in Fig. 1. For a strong 1D optical lattice there is no spatial overlap of wave functions of

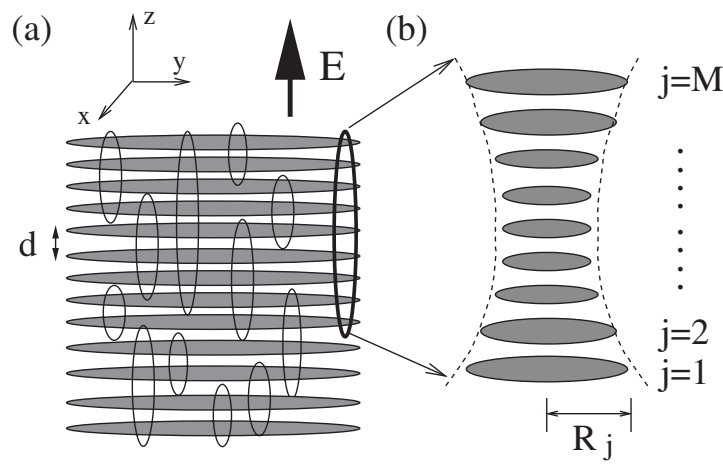

FIG. 1. (a) Schematic figure of a stack of pancake traps. The external electric field polarizes molecules in the direction perpendicular to the layers. Vertical ellipses denote self-assembled chains of molecules. (b) A typical shape of a dipolar chain. Shaded ellipses denote single molecule wave functions in individual layers [see Eq. (1)] with radius $R_{j}$. 


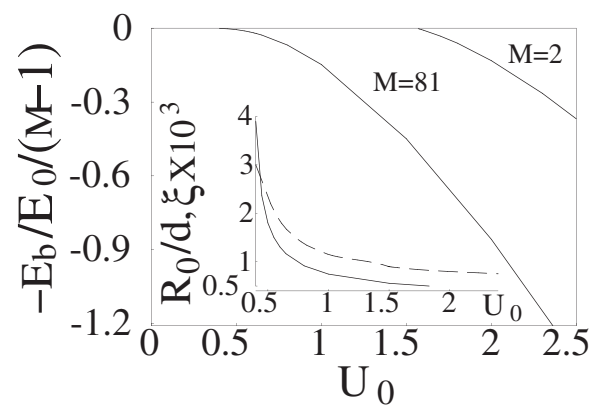

FIG. 2. Binding energy (note the minus sign) per molecule as a function of dipole strength, $U_{0}$, for chain length $M=2$ and $M=$ 81. Results are obtained using the variational wave function in Eq. (1), with $\omega=0.01 E_{0} . E_{0} \equiv \hbar^{2} / m d^{2}=\frac{2}{\pi^{2}} E_{R}$ with $E_{R}$ being the recoil energy of the $1 \mathrm{D}$ optical lattice. Inset: Optimal values of the variational parameters for a single chain, $R_{0}$ (solid curve) and $\xi$ (dashed curve), as a function of $U_{0}$ for $M=81$. Note that the value of $\xi$ has been multiplied by $10^{3}$ to fit the scale of the vertical axes.

molecules localized in different layers, hence $M$ dipoles in successive layers can be described by the Hamiltonian

$$
H_{M}=\sum_{j=1}^{M}\left[\frac{\mathbf{p}_{j}^{2}}{2 m}+\frac{m \omega^{2}}{2} \mathbf{r}_{j}^{2}\right]+\frac{1}{2} \sum_{i \neq j}^{M} V_{|i-j|}\left(\left|\mathbf{r}_{i}-\mathbf{r}_{j}\right|\right) .
$$

Here $V_{j}(\mathbf{r})=D^{2}\left(|\mathbf{r}|^{2}-2 j^{2} d^{2}\right) /\left(|\mathbf{r}|^{2}+j^{2} d^{2}\right)^{5 / 2}$ is the dipole-dipole interaction with $D$ being the electric dipole moment. $\omega$ is the in-plane trapping frequency and $m$ is the mass of a single molecule. When $L$ is the total number of layers in the 1D optical lattice, we can have $M=$ $2,3, \ldots, L$ as possible chain lengths. For simplicity, we assume that all layers are equivalent and contain the same number of molecules. We define new variables: $\mathbf{q}_{j} \equiv$ $\mathbf{p}_{j}-\mathbf{P} / M$ and $\mathbf{s}_{j} \equiv \mathbf{r}_{j}-\mathbf{R}$, where $\mathbf{P} \equiv \sum_{j=1}^{M} \mathbf{p}_{j}$ and $\mathbf{R} \equiv$ $\frac{1}{M} \sum_{j=1}^{M} \mathbf{r}_{j}$ are the total momentum and the center-of-mass (c.m.) position of the chain. As a result, $H_{M}$ can be separated into the sum of the c.m. part $\left(H_{\text {c.m. }}=\frac{\mathbf{P}^{2}}{2 M m}+\right.$ $\left.\frac{1}{2} M m \omega^{2} \mathbf{R}^{2}\right)$ and the part that describes the relative motion inside the chain:

$$
H_{\mathrm{rel}}=\sum_{j=1}^{M}\left[\frac{\mathbf{q}_{j}^{2}}{2 m}+\frac{m \omega^{2} \mathbf{s}_{j}^{2}}{2}\right]+\frac{1}{2} \sum_{i \neq j}^{M} V_{|i-j|}\left(\left|\mathbf{s}_{i}-\mathbf{s}_{j}\right|\right) .
$$

The commutation relation between the new coordinates are $[\mathbf{R}, \mathbf{P}]=i \hbar,\left[\mathbf{s}_{j}, \mathbf{q}_{l}\right]=i \hbar \delta_{j l}\left(1-M^{-1}\right)$, and all other commutators are zero. As we demonstrate below, important properties of a DCL are dominated by the long chains $(M \gg 1)$. So from now on we will treat $\mathbf{s}_{i}, \mathbf{q}_{j}$ as canonically conjugate variables for chains of all lengths. To calculate the binding energy from $H_{\text {rel }}$, we take the following variational wave function

$$
\psi_{M}\left(\left\{\mathbf{s}_{j}\right\}\right)=\prod_{j=1}^{M} \frac{\exp \left(-\left|\mathbf{s}_{j}\right|^{2} / 2 R_{j}^{2}\right)}{\sqrt{\pi} R_{j}},
$$

where the wave function width, $R_{j}$, is determined by the competition of the attractive part of dipole interaction, which favors localized wave functions, and the kinetic energy, which opposes localization. Near the chain center interaction has contributions from both sides of the chain, while near the chain tips interaction comes from one side only and is weaker. Therefore we expect a smaller size of the wave function near the center compared to the tips of the chain [see Fig. 1(b)]. For simplicity we assume $R_{j}=$ $R_{0}\left(1+\xi|j-(M+1) / 2|^{2}\right)$, where $R_{0}$ and $\xi$ are two variational parameters to be obtained by minimizing $\left\langle\psi_{L}\left(\left\{\mathbf{s}_{j}\right\}\right)\left|H_{\text {rel }}\right| \psi_{L}\left(\left\{\mathbf{s}_{j}\right\}\right)\right\rangle$. Taking more sophisticated trial wave functions will not have a qualitative effect on the properties of DCL that we discuss in this Letter.

In Fig. 2 we compare binding energies (per molecule) of the shortest $(M=2)$ and longest $(M=81)$ chains in a system with $L=81$ layers. The control parameter is the dimensionless strength of the dipole interaction, $U_{0} \equiv$ $m D^{2} / \hbar^{2} d$, which can be tuned either by increasing the electric field (which increases the value of dipole moments of molecules), by changing the interlayer distance $d$, or by increasing the effective mass of molecules using an additional in-plane optical lattice. A nontrivial result of our analysis is that bound states of many molecules (long chains) appear first and have a stronger binding energy. In the system under consideration, the longest chain $(M=$ $L=81$ ), can exist when $U_{0}$ exceeds a critical value $U_{0, L}^{*} \sim$ 0.4 . A bound state of two molecules appears only for $U_{0}>$ $U_{0,2}^{*} \sim 1.6$ [12]. So for $U_{0}<U_{0, L}^{*}$ no chains are formed, for $U_{0}>U_{0,2}^{*}$ chains of all lengths are stable, and for intermediate dipole strengths $\left(U_{0, L}^{*}<U_{0}<U_{0,2}^{*}\right)$ there is a critical value of the chain length $M_{0}$ such that chains of length $M_{0}$ or larger are stable but shorter chains are not (except for the unbound dipoles, $M=1$ ). In the inset of Fig. 2, we show the optimal values of the variational parameters, $R_{0}$ and $\xi$, for the longest chain $(M=81)$ obtained as a function of $U_{0}>U_{0, L}^{*}$. Our results show that when $U_{0}$ is small (weakly bound chains), the minimum width of the chain can be several times larger than the interlayer distance, $d$. In this limit the curvature of the shape $\xi$ is large and the chain has a shape of a horn in both ends [Fig. 1(b)]. For large dipole strengths we find tightly bound chains in which both $R_{0}$ and $\xi$ are very small.

We now proceed to discuss the thermodynamics of DCLs. We start by making several simplifying assumptions. Specifically, we neglect repulsive interactions between chains and do not include bending of individual chains. The first assumption is justified rigorously in the limit of the small number of molecules within each layer. For $N$ molecules in one layer, the characteristic strength of the in-plane dipolar energy is $E_{\mathrm{int}} \sim N D^{2} / a_{\mathrm{osc}}^{3}$, where $a_{\mathrm{osc}}=(\hbar / m \omega)^{1 / 2}$ is the in-plane oscillator length. We can neglect $E_{\text {int }}$ compared to $\hbar \omega$ when $N^{2} \omega<$ $\left(\hbar^{5} / D^{4} m^{3}\right)^{1 / 2}$. We further note that most conclusions involving thermodynamic properties of DCL will remain 
qualitatively similar even when interactions between chains become important so long as they are not strong enough to lock molecules within the planes into a Wigner crystal phase [6]. Neglecting bending of individual chains can be justified when temperatures are smaller than the characteristic binding energy and the chain lengths are not too large. This assumption may not necessarily apply near the condensation temperature for modest dipolar moments (see Fig. 3 and discussion below) but should be valid for large values of $U_{0}$ [13].

When the interactions between chains are neglected, the total energy of a DCL is a sum of energies of individual chains. Neglecting the bending modes, we can write the single chain energy as a sum of the center-of-mass and binding energies: $\varepsilon_{M, \mathbf{i}}=\hbar \omega\left(i_{x}+i_{y}+1\right)-E_{b, M}$. Here, $\mathbf{i}=\left(i_{x}, i_{y}\right)$ are quantum numbers for the c.m. coordinate in a $2 \mathrm{D}$ harmonic trapping potential and $E_{b, M}$ is the binding energy of a chain of length $M$. To understand thermodynamics of the DCL we need to sum over all chain configurations with the constraint that the total number of dipolar molecules is fixed. We enforce the constraint using the Lagrange multiplier method and obtain the partition function $Z$,

$$
\log Z=-\sum_{M=1, M_{0}}^{L} \sum_{\mathbf{i}} \sum_{\alpha_{M}} \log \left[1-e^{-\beta\left(\varepsilon_{M, \mathbf{i}}-M \mu\right)}\right] .
$$

Here $\beta=1 / k_{B} T$ and index $\alpha_{M}=\frac{M+1}{2}, \cdots, L-\frac{M-1}{2}$ counts possible c.m. positions of chains in the $z$ direction. In the above partition function the chemical potential term $M \mu$ is proportional to the chain length $M$. This follows from the fact that the numbers of chains are not fixed but

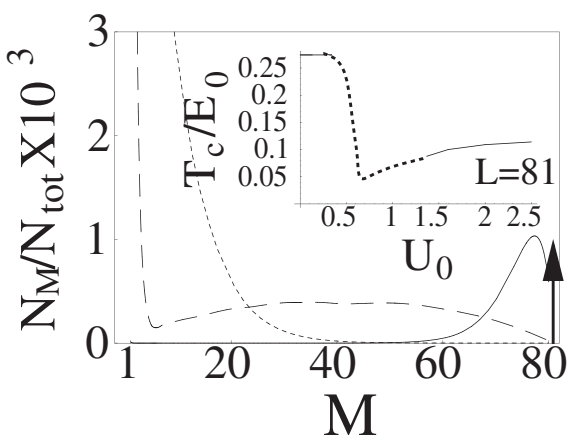

FIG. 3. Number of chains in a multilayer system $(L=81)$ as a function of the chain length, $M$. Other parameters are $N_{\text {tot }}=$ $10^{5}, U_{0}=2$, and $\hbar \omega=0.01 E_{0}$. Condensate $T_{c}$ is $0.11 E_{0}$ for such parameters. Solid, dashed, and dotted lines are for temperature $T=0.1,0.3$, and $0.5 E_{0}$, respectively, and the upward arrow indicates the condensation of the longest chain $(M=L=$ 81) Inset: Condensate $T_{c}$ as a function of dipole strength, $U_{0}$, for multilayer system $L=2$ and $L=81$. The number of dipoles per layer for these two systems are set the same so that they have the same $T_{c}$ in the noninteracting regime. Solid lines are calculated within the noninteracting chain approximation (discussed in the text). Dotted lines are schematic since reliable theory in the vicinity of $U_{0, L}^{*}$ should include interactions between chains. can change in disassociation- or association-type processes even in equilibrium. The only physical chemical potential $\mu$ is that of individual molecules and controls the distribution of chain lengths in thermal equilibrium. This is similar to well-known systems of multi-ionized plasmas [14] and classical rheological fluids [9]. To find $\mu$ in (2) we relate it to the total number of molecules $N_{\text {tot }}=$ $k_{B} T \partial \ln Z / \partial \mu=\sum_{M=1, M_{0}}^{L} M N_{M}$, where $N_{M}$ is the number of chains of length $M$. At temperatures above BoseEinstein condensation we have

$$
N_{M}(\mu)=(L-M+1)\left(\frac{k_{B} T}{\omega}\right)^{2} L i_{2}\left(z_{M}\right) .
$$

Here $z_{M} \equiv \exp \left[\beta\left(E_{b, M}+M \mu-\hbar \omega\right)\right]$ is the fugacity of chains of length $M$, and $L_{2}(z) \equiv \sum_{k=1}^{\infty} \frac{z^{k}}{k^{2}}$ is the polylogarithm function. In deriving expression (3) we replaced summation over discreet levels of the in-plane trap by the integration and used the identity, $\int_{0}^{\infty} \int_{0}^{\infty}\left[z^{-1} e^{x+y}-\right.$ $1]^{-1} d x d y=L i_{2}(z)$. The prefactor, $(L-M+1)$, counts the number of possible c.m. positions in the $z$ direction. Bose-Einstein condensation means the appearance of a macroscopic occupation of some microscopic state. In our case this happens when the argument inside the log of Eq. (2) goes to zero for some particular value of the quantum numbers $M$ and $\mathbf{i}$. From the dependence of the binding energy $E_{b, M}$ on the chain length $M$, one can see that the condensation occurs for the longest chains, $M=$ $L$, in the state $i_{x}=i_{y}=0$. To find the transition temperature we need to solve the equation $\mu\left(T_{c}\right)=L^{-1}(\hbar \omega-$ $\left.E_{b, L}\right) \equiv \mu_{c}$. For temperatures below $T_{c}$, the chemical potential stays fixed at $\mu_{c}$ and the number of chains in the condensate is given by the equation $N_{c}=L^{-1}\left[N_{\text {tot }}-\right.$ $\left.\sum_{M=1, M_{0}}^{L-1} M N_{M}\left(\mu_{c}\right)\right]$.

In Fig. 3 we show the calculated number of chains, $N_{M}$, as a function of their length for different temperatures. We choose $U_{0}=2.0$, which is large enough to allow formation of the chains of all length (see Fig. 2). At high temperatures (dotted line), however, the entropy is more important, so short chains and unbound molecules dominate. One can show that in this limit the distribution function obeys the Saha's equation that was originally derived for multiionized plasma [14]

$$
N_{M} \sim\left(\frac{N_{\mathrm{tot}}}{L}\right)^{M}\left(\frac{\hbar \omega}{k_{B} T}\right)^{2 M-2} e^{-E_{b, M} / k_{B} T} .
$$

As the temperature is lowered to be of the same order as the binding energy, the competition between entropy and energy leads to a nonmonotonic distribution of $N_{M}$ (dashed line), which is a special feature of the DCL approaching the regime of quantum degeneracy. When the temperature is reduced further (solid line), quantum statistics becomes important and we find a strong enhancement of the population of longer chains and eventually Bose-Einstein condensation of the longest chains. 
In the inset of Fig. 3, we show the condensate $T_{c}$ as a function of the dipole strength for a multilayer $(L=81)$ system. Solid lines in the $U_{0} \rightarrow 0$ regime are obtained treating molecules as noninteracting particles. For large $U_{0}$ regime we include self-assembly of molecules into chains but neglect interactions between chains. In the intermediate regime of $U_{0}$ (around the critical dipole strength, $U_{0, L}^{*}$ ), we expect the approximation of noninteracting chains to break down due to a larger transverse size of the chains (see the inset of Fig. 2). We use dotted lines to schematically describe the expected results of $T_{c}$ in this regime. We anticipate a sharp kink in the transition temperature in analogy to mixtures of bosonic atoms near the Feshbach resonance regime [15], due to the formation of real space bound states (chains) at $U_{0, L}^{*}$. We point out that $T_{c}$ is strongly suppressed in a multilayer system for $U_{0} \sim$ $U_{0, L}^{*}$ because the number of the longest chains is very small. This is a special feature of dipolar chain liquid, since in this regime binding energies of the longest chain are small so that molecules are spread out among chains of various lengths.

We now discuss experimental issues related to the realization and detection of the dipolar chain liquid proposed in this Letter. For typical polar molecules with electric dipole moment $D \sim 1$ Debye, $m \sim 100$ atomic mass unit, and trapped in the optical lattice of Nd: YAG laser $(d=$ $\lambda / 2=0.53 \mu \mathrm{m})$, we find the dimensionless dipole strength, $U_{0} \equiv m D^{2} / \hbar^{2} d$, is about 2.8 , and exceeds the critical dipole strength needed for the formation of dipolar chains (see Fig. 2). $U_{0}$ can be further controlled by an external dc electric field $\left(E_{\mathrm{dc}}\right)$. For example, by starting from a $\mathrm{BEC}$ of the nonpolarized molecules at $E_{\mathrm{dc}}=0$ one can drive a system into a DCL state by adiabatically increasing electric field. The resulting dipolar chain liquid can be observed by several different methods. For example, the frequencies of collective modes that are inhomogeneous along the stack will change dramatically for $U_{0}>U_{0, L}^{*}$, due to the large change in the stiffness of the dipolar cloud associated with the emergence of long chains. Likewise, $\mathrm{rf}$ spectroscopy can be used to probe the energy gap resulting from the chain formation. Successive absorption peaks shall be observable in the excitation spectrum, yielding a signature of chaining effect in the dipolar cloud. Finally, a long-range density correlation between dipoles within the chains can be measured via noise correlation techniques after the cloud is released from the in-plane trap.

We now discuss some novel avenues opened by this work. First, starting with a fixed number of layers and approaching the thermodynamic limit by increasing transverse system size, we expect that there will be a phase transition between a simple superfluid and a DCL at $U_{0}=$ $U_{0, L}^{*}$. Second, by increasing the dipole strength even further, a first order transition from superfluid of chains to a Wigner crystal phase should occur. In the Wigner crystal phase chains should form a triangular lattice with a fixed distance between them [6]. Finally, when considering bending and/or vibration of long chains at finite temperatures, we expect the DCL to be similar to a classical ferroliquid with nontrivial thermodynamic properties. We expect that each of these avenues correspond to an intriguing direction for future theoretical and experimental research.

In summary, we have predicted the chaining effect of polar molecules confined in a 1D optical lattice. The ground state and thermodynamic properties of the system can be qualitatively changed by the formation of chains. These intriguing phenomena should be observable in the experiments with ultracold polar molecules.

We acknowledge useful discussion with D. S. Fisher, D. DeMille, D. Nelson, and Ite Yu. This work was supported by NSC (D. W. W.), NSF (M.L.), NSF Grant No. DMR0132874 (E. D.), and CUA.

[1] J. Stuhler et al., Phys. Rev. Lett. 95, 150406 (2005).

[2] J. Doyle, Eur. Phys. J. D 31, 149 (2004); J. D. Weinstein et al., Nature (London) 395, 148 (1998); H. L. Bethlem et al., Nature (London) 406, 491 (2000); A. J. Kerman et al., Phys. Rev. Lett. 92, 033004 (2004).

[3] J. Stuhler et al., Phys. Rev. Lett. 95, 150406 (2005).

[4] L. Santos et al., Phys. Rev. Lett. 85, 1791 (2000); L. Santos et al., Phys. Rev. Lett. 90, 250403 (2003); Duncan H.J. O’Dell et al., Phys. Rev. Lett. 92, 250401 (2004).

[5] K. Góral et al., Phys. Rev. Lett. 88, 170406 (2002); R. Barnett et al., Phys. Rev. Lett. 96, 190401 (2006); D. DeMille, Phys. Rev. Lett. 88, 067901 (2002); A. Micheli, Nature Phys. 2, 341 (2006); R. B. Diener and T.-L. Ho Phys. Rev. Lett. 96, 190405 (2006).

[6] H. P. Buchler et al., cond-mat/0607294.

[7] P. M. Lushnikov, Phys. Rev. A 66, 051601 (2002);

[8] Z. Hadzibabic et al., Nature (London) 441, 1118 (2006); Z. Hadzibabic et al., Phys. Rev. Lett. 93, 180403 (2004).

[9] P. G. De Gennes and P. A. Pinous, Phys. Kondens. Mater. 11, 189 (1970); R. E. Rosensweig, Ferrohydrodynamics (Adademic, New York, 1985); P. I.C. Teixeira et al., J. Phys. Condens. Matter 12, R411 (2000).

[10] J. J. Weis and D. Levesque, Phys. Rev. Lett. 71, 2729 (1993); P. J. Camp et al., ibid. 84, 115 (2000); A.-P. Hynninen and M. Dijkstra, ibid. 94, 138303 (2005).

[11] V.F. Puntes et al., Science 291, 2115 (2001); M. Klokkenburg, Phys. Rev. Lett. 96, 037203 (2006).

[12] We note that the critical dipole strength for two layer $(M=2)$ can be rigorously calculated. Our calculation shows that $U_{0,2}^{*} \sim 0.71$, about 2 times smaller than the result obtained by variational approach.

[13] We expect that including bending modes would favor the longest chains even more by introducing physically distinct states, leading to a further reduction of $T_{c}$.

[14] L. D. Landau and E. M. Lifshitz, Statistical Physics (Pergamon, New York, 1973), 3rd ed.

[15] M. W. J. Romans et al., Phys. Rev. Lett. 93, 020405 (2004). 